\title{
-Mini Review- \\ Trials for Improvement of Blastocyst Stage Transfer Technique
}

\author{
Yoshiharu Morimoto $^{1 *}$ and Takuji Nishihara ${ }^{1}$ \\ ${ }^{1}$ The Centre for Reproduction and Infertility, IVF OSAKA CLINIC 2-2-30, Nagata-Naka, Higashi- \\ Osaka, Osaka 577-0013, Japan
}

Recently the blastocyst stage transfer (BST) has been applied with several kinds of sequential media and good results have been achieved. The technique made it possible to select embryo potential for implantation and to prevent multiple pregnancies, especially triplets. Several reports [1] have been published on the efficacy of BST. One paper reported an improved pregnancy rate compared with Day 3 transfer and another [2] reported a very high conception rate and statistically better results than Day 2/3 transfer in cases of recurrent failure and aged women [3]. Several studies have described, however, the rate of conception to be approximately equal compared to that of the conventional method $[4,5]$. It would be therefore important to choose cases to apply BST to, such as recurrent unsuccessful or aged cases.

Since BST has been developed, most IVF centers have adopted the technique, but there would be more room to improve the clinical results.

In the present study, the choice of the medium in BST was discussed. And the efficacy of the two-step embryo transfer method that is one of the techniques developed to apply BST was estimated.

\section{Choice of Medium}

Firstly a comparison of Cleavage medium and Blastocyst medium (COOK IVF Co. LTD, Australia; COOK group) and IVC-1 and IVC-2 (In Vito Care, Co.Itd U.S.A; IVC group) was done. The parameters were estimated with COOK medium in 71 periods and IVC medium in 48 periods of treatment cycles from October 2001 to March 2002. Ovarian stimulation was performed by a long or ultrashort protocol. Follicle stimulating hormone or human menopausal

Received: January 12, 2003

Accepted: February 10, 2003

*To whom correspondence should be addressed. gonadotrophin was administered from Day 3 and oocytes were picked up 36 hours after an injection of hCG $5000 \mathrm{IU}$ in the $20 \mathrm{~mm}$ diameter follicles. Conventional IVF or ICSI was selected according to the condition of the semen.

HTF (Irvine Scientific, Co. LTD, U.S.A.) medium supplemented with $10 \%$ SSS was used for insemination and after ICSI. One milliliter of HTF (Irvine Scientific, Co. LTD, U.S.A.) medium supplemented with $10 \%$ SSS in a NUNC four-well dish was used for insemination after ICSI, and the medium was changed to IVC-1 or Cleavage medium, respectively, after a fertilization check on Day 1 and to IVC-3 and the Blastocyst medium on Day 3. Embryos were observed only on Days 3 and 5 . If only morula was acquired on Day 5, they were cultured until the next day and transferred at the blastocyst stage in the case of successful blastocyst achievement.

The background of each case is shown in Table 1. There was no difference between the groups in age, number of IVF/ICSI trials or the number of blastocyst transferred. The rate of blastocyst formation was $44.7 \%$ in the COOK group and $43.4 \%$ in the IVC group per cycle and $43.8 \%$ in the COOK group and $42.7 \%$ in the IVC group per embryo. There was no significant difference between the result for two kinds of medium. The pregnancy rate was $38.7 \%(24 / 62)$ in the COOK group and $40.0 \%(18 / 45)$ in the IVC group, there being no statistically significant difference. Similarly, there was no difference between the cancellation rates (12.7\% and $6.3 \%$ ) (Table 2).

Next, a comparison was made between IVC-1, IVC-2 and Medium 1 and 2 (Medicult Co.LTD, Denmark). Thirty-five cases in which at least more than eight oocytes were obtained and it was planned to be used in BST were selected as materials. All oocytes in each case were divided into two groups and used for each medium. 
Table 1. Clinical background of experiment 1

\begin{tabular}{lccc}
\hline & COOK & IVC & P value $^{\mathrm{a}}$ \\
\hline No. of periods & 71 & 48 & $\mathrm{NS}$ \\
Age & $36.2 \pm 3.7$ & $37.2 \pm 4.1$ & $\mathrm{NS}$ \\
No. of IVF trial & $3.1 \pm 2.0$ & $2.9 \pm 1.9$ & $\mathrm{NS}$ \\
No. of retrieved oocyte & $9.2 \pm 6.2$ & $9.5 \pm 6.6$ & $\mathrm{NS}$ \\
Fertilization rate (\%) & $83.5 \pm 25.6$ & $77.8 \pm 23.0$ & $\mathrm{NS}$ \\
Endometrial thickness (mm)* & $10.9 \pm 2.1$ & $10.7 \pm 2.0$ & $\mathrm{NS}$ \\
No. of transferred embryo & $1.8 \pm 1.9$ & $1.7 \pm 1.0$ & $\mathrm{NS}$ \\
\hline & \multicolumn{3}{c}{ (mean \pm SD) } \\
a: Student's $t$-test. *Endometrial thickness on the day of embryo transfer.
\end{tabular}

Table 2. Blastocyst formation rate and pregnancy rate in experiment 1

\begin{tabular}{llcc}
\hline & COOK & IVC & P value \\
\hline Blastocyst formation rate* & $44.7 \pm 29.0$ & $43.4 \pm 34.5$ & $\mathrm{NS}^{\mathrm{a}}$ \\
Blastocyst formation rate** & $43.8(159 / 363)$ & $42.7(144 / 337)$ & $\mathrm{NS}^{\mathrm{b}}$ \\
Pregnancy rate per period & $33.8(24 / 71)$ & $37.5(18 / 48)$ & $\mathrm{NS}^{\mathrm{b}}$ \\
Pregnancy rate per transfer & $38.7(24 / 62)$ & $40.0(18 / 45)$ & $\mathrm{NS}^{\mathrm{b}}$ \\
Cancellation rate of ET & $12.7(9 / 71)$ & $6.3(3 / 48)$ & $\mathrm{NS}^{\mathrm{b}}$ \\
\hline & \multicolumn{3}{r}{ (mean $\pm \mathrm{SD}, \%)$} \\
a. Student's $t$-test. ${ }^{\text {b }: ~} \chi^{2}$ test. *Average blastocyst formation rate in each period. \\
**Blastocyst formation rate of all embryos.
\end{tabular}

Several parameters such as embryo growth at Day 3, the rate of blastocyst formation, the morphology of blastocysts, pregnancy rate and implantation rate were estimated.

The culture up to the blastocyst stage was done by the same method as in the foregoing experiment.

There was no significant difference between the two groups in the number of blastomere and in the embryo morphology at Day 3, and there was no difference in the rate of blastocyst formation. The grade score for blastocysts calculated on the basis of the method of Gardner at Day 5 was significantly better in the Medicult group (Fig. 1).

The overall pregnancy rate was $45.7 \%(16 / 35)$ and the implantation rate was $30.2 \%(23 / 76)$ in 35 periods. The pregnancy rate and implantation rate were $23.1 \%$ and $22.2 \%$ in the IVC group (cultured only in IVC medium), $45.5 \%$ and $31.8 \%$ in the IVC and Medicult mixed group and $72.7 \%$ and $37.0 \%$ in the MediCult group (cultured only in MediCult medium). The pregnancy and implantation rates were significantly higher in the Medicult group than in the IVC group (Table 3).

\section{Effectiveness of Two Step Embryo Transfer}

Recently the concept of a receptivity induction mechanism of the embryo to implantation has been described, and a high rate of pregnancy has been reported in a two-step transfer method applying this concept [10]. In this method second and third quality embryos are transferred at Day 2 and the best quality embryos reaching the blastocyst are transferred at Day 5. We apply this method to cases in which success was not acquired even if advanced good quality blastocyst was transferred, because it seemed likely to be due to the lack of ability of receptivity in endometrium. It could be expected to reduce the cancellation rate in two-step embryo transfer more than that in BST. In this method the selection of embryos for longer culture would be essential. The rate of formation rate to the blastocyst stage was high in embryos over grade 3 and over 7 cells at Day 3 in our study. We therefore selected embryos with fewer than $30 \%$ fragmentation and with rapid growth for BST.

This method was applied to 77 cases in 83 periods. The average IVF/ICSI trial before two-step embryo transfer was 3.5 times and the average numbers of retrieved eggs and the fertilization rate were 11.7 oocytes and $86.4 \%$. And $29.6 \%$ of embryos reached 

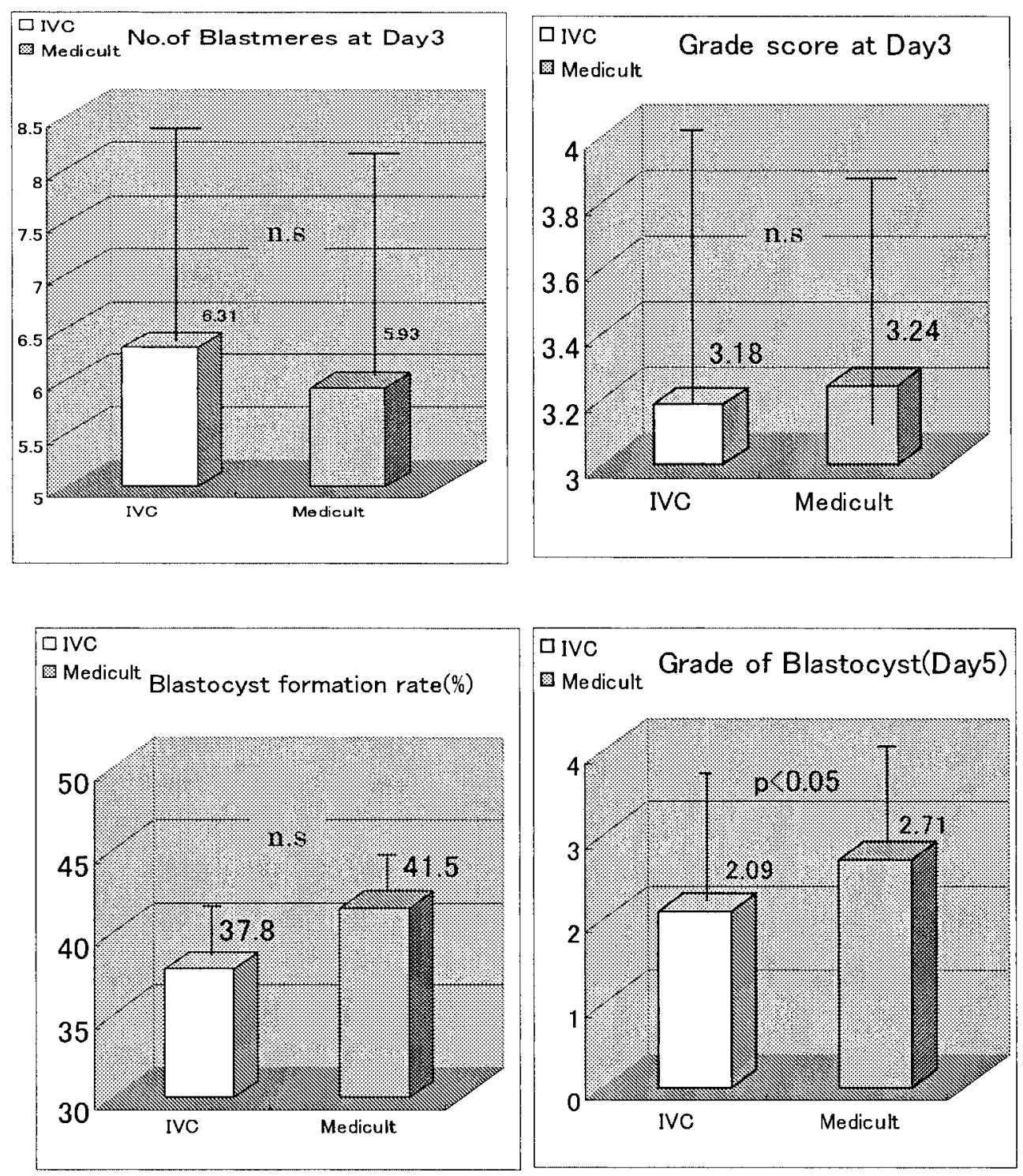

Fig. 1. Quality and cleavage speed of embryos at Day 3 and Day 5. Comparison of each parameters between the IVC and the MediCult group. (Grade scores were calculated based on the method of Gardner. Each point from one to five was assigned to each grade.)

the blastocyst stage and pregnancy and implantation rate were $40.7 \%$ and $16.6 \%$, respectively. Moreover those two parameters were $27.0 \%$ and $14.1 \%$ even in the limited number of cases with more than 4 failures (Table 4). The cancellation rate $(2.4 \%)$ was lower than in BST as already mentioned.

\section{Conclusion and Discussion}

One new medium for BST has been developed after another, and, fortunately many kinds of medium are commercially available now, so that higher quality blastocysts have consequently been acquired. Each medium has its characteristics and it is important to choose from the point of view of not only the rate of achievement to blastocysts, but the acquirement of growing potential to a fetus. Some reports have described embryo morphology on Day 3 rather than that of blastocysts as related to the success rate $[6,7]$ and the selection of the medium should be taken into consideration of the condition of the embryo at Day 3.

We have noticed the action of Glutathionine which is 
Table 3. Pregnancy rate and implantation rate in each group in experiment 2

\begin{tabular}{llcc}
\hline & IVC only & IVC+MediCult & Medicult only \\
\hline No. of periods & 13 & 11 & 11 \\
Pregnancy rate (\%)* & $23.1(3 / 13)^{\mathrm{a}}$ & $45.4(5 / 11)$ & $71.7(8 / 11)^{\mathrm{b}}$ \\
Implantation rate (\%) & $22.2(6 / 27)$ & $31.8(7 / 22)$ & $37.0(10 / 27)$ \\
\hline
\end{tabular}

${ }^{\mathrm{a}, \mathrm{b}} \mathrm{P}<0.05, \chi^{2}$ test. ${ }^{*}$ Pregnancy rate per period and transfer (no cancellation case).

Table 4. Result of two step embryo transfer method

\begin{tabular}{lcc}
\hline & All cases & Multiple failure cases* \\
\hline No. of cases & 77 & 32 \\
No. of periods & 83 & 36 \\
No. of IVF Trial & $3.5 \pm 2.2$ & $5.4 \pm 1.9$ \\
No. of retrieved oocytes & $11.7 \pm 5.4$ & $11.3 \pm 5.4$ \\
Fertilization rate (\%) & $86.4 \pm 14.1$ & $85.0 \pm 13.7$ \\
Blastocyst formation rate (\%) & $29.6 \pm 19.6$ & $27.0 \pm 19.5$ \\
No. of transferred embryos & $2.8 \pm 0.5$ & $2.8 \pm 0.5$ \\
Endometrial thickness (mm)** & $11.0 \pm 2.3$ & $10.7 \pm 2.3$ \\
Pregnancy rate per transfer (\%) & $40.7(33 / 81)$ & $36.1(13 / 36)$ \\
Pregnancy rate per period (\%) & $39.8(33 / 83)$ & $36.1(13 / 36)$ \\
Implantation rate $(\%)$ & $16.6(38 / 229)$ & $14.1(14 / 99)$ \\
\hline
\end{tabular}

(mean $\pm \mathrm{SD})$

*In the case of more than four IVF failures. **Endometrial thickness on the day of embryo transfer.

related to the embryo growth and which is a constituent of IVC-1 medium (In Vitro Care, Co. LTD, USA). Glutathionine is thought to contribute to embryo development [8] to high quality blastocysts and a higher implantation rate.

In the present investigation, we achieved high pregnancy rates by using the medium of MediCult medium which resulted from several trials. However it is not clear which ingredient was responsible for increasing the potential for implantation. The optimal conditions for blastocyst culture include growth factors and cytokines which are arranged in vivo, but it should be necessary to decide on the positive addition of those components for the more development of new media.

The damage to embryos should be considered in the long culture and the problem of laboratory space might be involved in laboratory management. Moreover as care should be taken not to make cultures long in order to take advantage of ingredients in bioavailable forms such as in L-Glutamine, the laboratory work might be more complicated if it involves frequent medium change. We have tried to prepare the medium at night for the next morning to reduce time for equilibriation to the minimum. And we also tried to reduce the time for microscopical observation by checking only at Day 3 and Day 5 to maintain a better culture environment.

In this study there was no significant difference in the embryo development at Day 3 and achieved a uniform rate to the blastocyst stage in all medium groups, but there were morphologically better quality blastocysts obtained with the medium of MediCult medium, consequently there were higher pregnancy and implantation rates. Hence, it would be desirable to use such approaches as chromosomal analysis, not only from the morphological point of view.

It would be of great benefit to reduce the number of embryos in order to prevent multiple pregnancies especially triplets, by using this technique. Nevertheless it has such demerits as cancellation of embryo transfer in some cases. According to Alper [11], implantation might be achieved if an embryo in which cleavage is arrested before the blastocyst stage was transferred at the four to eight cell stage. Thereafter, it was concluded the relevant evaluation of the merits of this method is still difficult in spite of the wide application due to the progress of cryopreservation technique of blastocysts.

In the application of two step embryo transfer, success was achieved even in difficult cases with more than four recurrent failures. The usefulness of BST was 
reported based on the different origin. Fanchin [12] showed that contractility of the uterus had been reduced day by day after hCG administration and disappeared at Day 7 of blastocyst transfer. In view of this fact, it might be advantageous to transfer after long culture.

One report pointed out a relationship between the condition of sperm and blastocyst formation. Miller [13] has reported low development to blastocysts and poor quality of blastocysts observed in cases of impaired semen condition. Moreover, Balaban [14] reported a low rate of blastocyst formation and implantation in cases of non-obstructive azoospermic patients. Improvement of clinical results in BST could be expected if patients were selected on the basis of semen condition.

\section{References}

1) Milki, A.A., Hinckley, M.D., Fisch, J.D., Dasig, D. and Behr, B. (2000): Comparison of blastocyst transfer with day 3 embryo transfer in similar patient populations. Fertil Steril., 73, 126-129.

2) Gardner, D.K., Schoolcraft, W.B., Wagley, L., Schlenker, T., Stevens, J. and Hesla, J. (1998): A prospective randomized trial of blastocyst culture and transfer in invitro fertilization. Hum Reprod., 13, 3434-3440.

3) Iwamoto, H., Fukuda, A., Kawata, A., Yamasaki, M., Tohnaka, M., Nishihara, T., Nakaoka, Y., Morimoto, Y. and Kanzaki, H. (2002): The efficiency of blastocyst transfer and 2 step embryo transfer was evaluated for repeated IVF failures. Journal of Japan Society of Fertilization and Implantation, 19, 25-28.

4) Utsunomiya, T. (2001): Blastocyst stage transfer. Clinical Gynecology and Obstetrics, 54, 1339-1401.

5) Coskun, S., Hollanders, J., Al-Hassan, S., Al-Sufyan, H., Al-Mayman, H. and Jaroudi, K. (2000): Day 5 versus day 3 embryo transfer: a controlled randomized trial. Hum Reprod., 15, 1947-1952.

6) Rijnders, P.M. and Jansen, C.A. (1998): The predictive value of day 3 embryo morphology regarding blastocyst formation, pregnancy and implantation rate after day 5 transfer following in-vitro fertilization or intracytoplasmic sperm injection. Hum Reprod., 13, 2869-2873.

7) Sai, N.N., Goldstein, J., Rowland, D.Y. and Goldfarb, J.M. (2000): Morphological evaluation of human embryos and derivation of an embryo quality scoring system specific for day 3 embryos: a preliminary study. Hum Reprod., 15, 2190-2196.

8) Jeong, B.S. and Yang, X. (2001): Cysteine, glutathione, and Percoll treatments improve porcine oocyte maturation and fertilization in vitro. Mol Reprod Dev., 59, 330-335.

9) Boquest, A.C., Abeydeera, L.R., Wang, W.H. and Day, B.N. (1999): Effect of adding reduced glutathione during insemination on the development of porcine embryos in vitro. Theriogenology, 51, 1311-1319.

10) Goto, S., Takebayashi, K., Shiotani, M., Fujiwara, M., Hirose, M. and Noda, Y. (2003) Effectiveness of two-step (consecutive) embryo transfer: comparison with cleavagestage embryo transfer. J. Reprod. Med. (in press).

11) Alper, M.M., Brinsden, P., Fischer, R. and Wikland, M. (2001): To blastocyst or not to blastocyst? That is the question. Hum Reprod., 16, 617-619.

12) Fanchin, R., Ayoubi, J.M., Righini, C., Olivennes, F., Schonauer, L.M. and Frydman, R. (2001): Uterine contractility decreases at the time of blastocyst transfers. Hum Reprod., 16, 1115-1119.

13) Miller, J.E. and Smith, T.T. (2001): The effect of intracytoplasmic sperm injection and semen parameters on blastocyst development in vitro. Hum Reprod., 16, 918-924.

14) Balaban, B., Urman, B., Isiklar, A., Alatas, C., Mercan, R., Aksoy, S. and Nuhoglu, A. (2001): Blastocyst transfer following intracytoplasmic injection of ejaculated, epididymal or testicular spermatozoa. Hum Reprod., 16, 125-129. 